\title{
LAPAROSCOPIC INTRAPERITONEAL ONLAY MESH REPAIR OF INCISIONAL HERNIAS - A RETROSPECTIVE STUDY
}

Mumtaz Ahmad Ansari ${ }^{1}$

\section{HOW TO CITE THIS ARTICLE:}

Mumtaz Ahmad Ansari. "Laparoscopic Intraperitoneal Onlay Mesh Repair of Incisional Hernias - A Retrospective Study". Journal of Evolution of Medical and Dental Sciences 2014; Vol. 3, Issue 09, March 03; Page: 2323-2326, DOI: $10.14260 /$ jemds/2014/2142

ABSTRACT: BACKGROUND: Traditionally, ventral hernia repair has been done using preperitoneal mesh placement through an open technique. The laparoscopic technique is believed to have fewer complications and a better postoperative course, with quicker ambulation, shorter hospital stay, less postoperative pain and low recurrence rates. In this study, we have evaluated the laparoscopic technique and its outcome. METHODS: All patients presenting in our unit between March 2011 and March 2013 with a ventral hernia greater than $4 \mathrm{~cm}$ were planned for laparoscopic repair under general anesthesia. A polypropylene mesh was used as an intraperitoneal onlay mesh. A minimum overlap of the prosthesis of $3 \mathrm{~cm}$ in all directions was obtained. RESULTS: In 49 patients, out of 52 patients planned, laparoscopic repair was performed. Early postoperative complications were seen in nine patients $(18.4 \%)$ and late postoperative complications in three patients (6.1\%). CONCLUSION: Laparoscopic repair of incisional and ventral hernia is a safe alternative for open mesh repair. Further definition of indications is needed regarding size and localization of hernia.

KEYWORDS: Incisional Hernia, Mesh repair.

INTRODUCTION: Incisional hernias larger than $2 \mathrm{~cm}$ are preferably repaired using a prosthesis, because primary repair has an unacceptable high rate of recurrences ${ }^{1}$. Traditionally the prosthesis is placed in a preperitoneal position avoiding contact between the bowel and the prosthesis. Since a few years, prostheses are manufactured with two different surfaces. One side has a low tendency to form adhesions, and can therefore be placed in contact with the bowel. Laparoscopic technique to place an intraperitoneal mesh to cover the hernia defect (IPOM or Intra-Peritoneal Onlay Mesh) was first reported in 1993 by LEBLANC and BOOTH ${ }^{2}$. Several recent studies report on the safety and feasibility of laparoscopic repair of ventral hernia ${ }^{3-6}$. The laparoscopic technique is believed to have fewer complications and a better postoperative course, with quicker ambulation, shorter hospital stay and less postoperative pain. Low recurrence rates of 0 to $9 \%$ are reported ${ }^{1}$. This study was done to evaluate the results of our initial experience.

MATERIAL AND METHODS: All patients presenting between March 2011 and March 2013 in our unit with a ventral hernia greater than $4 \mathrm{~cm}$ were planned to have a laparoscopic repair. Operations were performed under general anesthesia with the patient supine and the arms at the side of the patient. Single dose of prophylactic antibiotic and Tetanus toxoid was administered preoperatively. A laparoscope of 5 or $10 \mathrm{~mm}$ was used. We used a polypropylene mesh as an intraperitoneal onlay mesh.

RESULTS: In 49 patients, out of 52 patients planned, laparoscopic repair was performed. Three patients were converted to laparotomy. Two because unexpected recurrence of malignancy was 
encountered in patients previously operated for malignancy. The operation was converted to an open oncological resection. In one patient with multiple previous laparotomies the adhesions were so dense that an adequate pneumoperitoneum could not be created, and the operation was converted to an open mesh repair after open adhesiolysis. Mean age of patients was 63 years (range, 40-88), with 25 males and 24 females. Obesity with body mass index $>30$ was present in $49 \%$ of the patients. The indication for surgery was an incisional hernia in 88\% and recurrence after open hernia repair in $43 \%$. Major reasons for consultation were pain, discomfort and anxiety about the growth of the hernia. Ten patients had symptoms of sub-obstruction or history of incarceration. In two patients the operation was performed in an emergency setting because of an incarcerated hernia that could not be reduced preoperatively. The hernia was located on the midline in $76 \%$. The others were either subcostal or in the iliac fossa. The mean hernia surface area was $86,6 \mathrm{~cm} 2$ and $43 \%$ had a width greater than $10 \mathrm{~cm}$. Table I classifies the patients according to the classification proposed by J. P. CHEVREL7.

A minimum overlap of the prosthesis of $3 \mathrm{~cm}$ in all directions was obtained. There were no intraoperative complications and the mean operating time was $103 \mathrm{~min}$. Postoperative complications were encountered in nine patients (18.4\%) (Table II). Mean hospital stay was 5, 9 days. Mean followup was 14.3 months. Late complications were seen in three patients (6.1\%). Two patients were reoperated for small bowel obstruction. In one patient (the patient who also had the recurrence) the obstruction was caused by adhesions to the margin of the prosthesis at the level of the spiral tacks; in one patient because of adhesions not related to the prosthesis. In one patient three transfascial sutures were removed under local anesthetics because of persisting localized pain at the site of these sutures.

DISCUSSION: Several large series on laparoscopic ventral hernia have been reported ${ }^{3-5}$. This technique has proven to be a safe and feasible alternative to open mesh repair ${ }^{1}$. Although many retrospective series are available, only one randomized trial on 60 patients comparing open versus laparoscopic mesh repair has been published by CARBAJO et al. ${ }^{8}$ They found that laparoscopic repair had a lower complication rate, shorter operating time, shorter hospital stay and lower recurrence rate at 27 months mean follow-up. Ventral hernias are a heterogeneous group with either primary or incisional hernias and different sizes and localizations of the hernias. Further work has to be done to define the best indications for the laparoscopic technique.

Midline juxtaumbilical incisional hernias of moderate size are probably the best indications for the technique.

In hernias extending to the suprapubic or the xyphoid region it is sometimes difficult to obtain a good overlap and fixation of the mesh. Our one recurrence so far was indeed a suprapubic hernia underneath the implanted prosthesis. Obesity is probably also a patient variable favoring laparoscopic technique because of the advantages derived from avoiding large incisions and dissections in these patients. Parastomal incisional hernias present specific problems. A defect in the prosthesis has to be created and this makes proper fixation of the mesh challenging.

Moreover the case load of parastomal hernias is low and therefore it is difficult to acquire experience. 
Concerning the size, no real upper limit has been defined yet. Very large hernias with important loss of abdominal wall substance, as seen after open abdominal treatment for peritonitis, will need other techniques to repair than intraperitoneal laparoscopic mesh repair.

Overlap of the mesh over the hernia defect of at least $3 \mathrm{~cm}$ is considered to be necessary as was described with the original technique 2 . In the latest reviews, an even larger overlap of 4 or $5 \mathrm{~cm}$ is promoted 6 . In our series we usually tailored the prosthesis to exactly $3 \mathrm{~cm}$ overlap.

Accidental enterotomy is a dangerous complication during laparoscopic ventral hernia repair. Certainly if it is not noticed during the operation it may cause severe postoperative septic complications ${ }^{6}$. Therefore it is important to have a meticulous technique for adhesiolysis.

Always search for the avascular plane between the adhesions and the abdominal wall or the hernial sac. Avoid the use of electrocautery as much as possible and perform adhesiolysis by sharp dissection of the adhesions rather than by traction on the bowel. Most challenging are adhesions to prosthetic material from previous open mesh repair, because there the avascular plane might not be present.

Postoperative complications ${ }^{\circ}$

Early postoperative complications:

Serious pain at fixation suture for $>6$ weeks 4

Symptomatic seroma $>6$ weeks 2

Pneumonia 2

Urinary retention 1

Prolonged hiccup 1

Bleeding 0

Wound infection 0

Mesh infection 0

Intra-abdominal sepsis or abscess 0

Prolonged ileus 0

Late postoperative complications

Hernia recurrence 1

Obstruction with reoperation 2

Chronic pain with removal of fixation sutures 1

Early postoperative complications were seen in nine patients $(18.4 \%)$ and late postoperative complications in three patients (6.1\%) of 49 patients who had a laparoscopic ventral hernia repair. 708 F. Muysoms et al.

CONCLUSION: Laparoscopic repair of incisional and ventral hernia is a safe alternative for open mesh repair. Further definition of indications is needed based on the dimension and the localization of the hernia. If the omission of transabdominal wall sutures, as has been proposed by some authors, improves the postoperative course with no adverse effect on recurrence rate, will be the subject of a randomized trial we have started this year. 


\section{ORIGINAL ARTICLE}

\section{REFERENCES:}

1. Cassar K., Munro A. Surgical treatment of incisional hernia. Br J Surg, 2002, 89: 534-45.

2. Leblanc K. A., Booth W. V. Laparoscopic repair of incisional abdominal hernias using expanded polytetrafluoroethylene: preliminary findings. Surg Laparosc Endosc, 1993, 3: 39-41.

3. Heniford B. T., Park A., Ramshaw B. J., Voeller G. Laparoscopic repair of ventral hernias: nine years' experience with 850 consecutive hernias. Ann Surg, 2003, 238: 391-400.

4. Carbajo M. A., Martin Del Olmo J. C., Blanco J. I. et al. Laparoscopic approach to incisional hernia. Lessons learned from 270 patients over 8 years. Surg Endosc, 2003, 17: 118-22.

5. Leblanc K. A., Whitaker J. M., Bellanger D. E., Rhynes V. K. Laparoscopic incisional and ventral hernioplasty: lessons learned from, 200 patients. Hernia, 2003, 7: 118-24.

6. Berger D., Bientzle M., Muller A. Postoperative complications after laparoscopic incisional hernia repair: incidence and treatment. Surg Endosc, 2002, 16: 1720-3.

7. Chevrel J. P. Classification of incisional hernia of the abdominal wall. In: Morales-Condé S, eds. Laparoscopic Ventral Hernia Repair. Barcelona: Springer, 2002: 65-72.

8. Carbajo M. A., Martin Del Olmo J. C., Blanco J. I. et al. Laparoscopic treatment vs. open surgery in the solution of major incisional and abdominal wall hernias with mesh. Surg Endosc, 1999, 13: 250-2.

\section{AUTHORS:}

1. Mumtaz Ahmad Ansari

\section{PARTICULARS OF CONTRIBUTORS:}

1. Associate Professor, Department of General Surgery, Institute of Medical Sciences, Banaras Hindu University.

\section{NAME ADDRESS EMAIL ID OF THE CORRESPONDING AUTHOR:}

Dr. Mumtaz Ahmad Ansari, Department of General Surgery, Institute of Medical Sciences, Banaras Hindu University. E-mail: mumtazbhu@gmail.com

Date of Submission: 06/02/2014. Date of Peer Review: 07/02/2014. Date of Acceptance: 13/02/2014. Date of Publishing: 28/02/2014. 